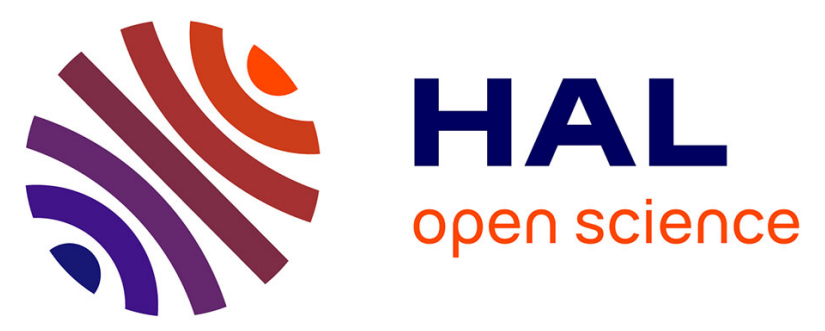

\title{
Structure of Metal-Organic Framework Glasses by Ab Initio Molecular Dynamics
}

\author{
Romain Gaillac, Pluton Pullumbi, Thomas D Bennett, François-Xavier
}

Coudert

\section{> To cite this version:}

Romain Gaillac, Pluton Pullumbi, Thomas D Bennett, François-Xavier Coudert. Structure of MetalOrganic Framework Glasses by Ab Initio Molecular Dynamics. Chemistry of Materials, 2020, 32 (18), pp.8004-8011. 10.1021/acs.chemmater.0c02950 . hal-02945127

\section{HAL Id: hal-02945127 \\ https://hal.science/hal-02945127}

Submitted on 22 Sep 2020

HAL is a multi-disciplinary open access archive for the deposit and dissemination of scientific research documents, whether they are published or not. The documents may come from teaching and research institutions in France or abroad, or from public or private research centers.
L'archive ouverte pluridisciplinaire HAL, est destinée au dépôt et à la diffusion de documents scientifiques de niveau recherche, publiés ou non, émanant des établissements d'enseignement et de recherche français ou étrangers, des laboratoires publics ou privés. 


\title{
Structure of Metal-Organic Framework Glasses
} by Ab Initio Molecular Dynamics

\author{
Romain Gaillac, ${ }^{\dagger} \ddagger$ Pluton Pullumbi, ${ }^{\ddagger}$ Thomas D. Bennett,, $\mathbb{I}$ and \\ François-Xavier Coudert*, $\dagger$ \\ $\dagger$ Chimie ParisTech, PSL Research University, CNRS, Institut de Recherche de Chimie Paris, \\ 75005 Paris, France \\ ¥Air Liquide, Centre de Recherche Paris Saclay, 78354 Jouy-en-Josas, France \\ IDepartment of Materials Sciences \& Metallurgy, University of Cambridge, Cambridge, UK \\ E-mail:fx.coudert@chimieparistech.psl.eu
}




\begin{abstract}
While metal-organic frameworks have been mostly studied in their crystalline form, recent advances have been made on their amorphous phases, both in fundamental understanding and in relation to possible applications. In particular, the zeolitic imidazolate (ZIF) glasses, that can be obtained from quenching liquid ZIFs, have shown promise. However, the details of their microscopic structure are very hard to probe experimentally. Here we use ab initio molecular dynamics simulations to investigate the nature of the ZIF glasses obtained from quenching molten ZIFs in silico. Through computational modeling of the melt-quench process on three different ZIF crystals, we aim to understand the effect of topology and chemistry upon the structure of the glass, compared to crystalline precursor and high temperature liquid. It is the first direct computational description of MOF glasses at the quantum chemical level. We find that both the chemical nature of the imidazolate linker and the topology of the framework play a role in the behaviour upon quenching, and the properties of the glasses obtained: structure, average coordination, and pore space.
\end{abstract}




\section{Introduction}

The discovery of three-dimensional coordination polymers, $\frac{112}{12}$ preceded the development of porous metal-organic frameworks (MOFs), which have now been intensely studied for 20 years, $\frac{344}{\text {. These }}$ compounds have so far essentially been considered in their crystalline state. ${ }^{[5}$ These extremely chemically versatile materials with regular three-dimensional frameworks can exhibit large specific surface areas and pore volumes, and have thus been proposed for applications in adsorption and catalysis, ${ }^{6}$ such as the capture of carbon dioxide ${ }^{7}$ or the catalytic degradation of harmful substances. ${ }^{[8}$ Furthermore the crystalline nature of many MOFs facilitates accurate and detailed structural determination by X-ray crystallography possible. However, more and more MOFs are shown to present disordered structures: this includes frameworks that contain a significant concentration

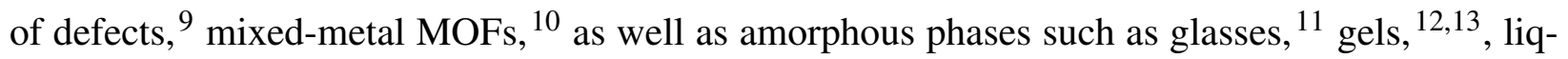
uids,, 14 and composites thereof. ${ }^{[15}$ Such materials can present interesting physical and chemical properties, such as improved mechanical and thermal stability. 16 Amorphous MOFs have been proposed for various applications based on their specific properties, such as controlled release, 17 irreversible trapping of harmful substances, $\frac{18}{18}$ or optically active glass-like materials $\frac{19}{19}$. In particular, amorphous MOF gels and glasses have recently gained interest in niche electronic applications where enhanced flexibility, transparency, luminescence switching and high charge mobility can be achieved by using thin film deposition and controlling the amorphous to crystalline transition of the deposited layer. ${ }^{20}$ Experimentally, these disordered framework structures can be obtained by many different physical or chemical means, including by direct synthesis 21 , by melting and quenching crystalline MOFs, $\frac{11}{1}$ through the application of mechanical pressure, $\stackrel{22}{21}$ or by ball milling 23 .

The determination of the microscopic structure and properties of amorphous phases of MOFs is, compared to their crystalline counterparts, vastly more difficult. For structural characterization, authors have reported the use of variable temperature X-ray and neutron pair distribution function experiments, which are often the only techniques that can offer direct insight at the microscopic level. By using this data and applying reverse Monte Carlo (RMC) modelling, it is possible to generate models of the disordered phase. ${ }^{24|25|}$ However, the models obtained in this way typically 
depend largely on the initial configuration chosen (e.g., using an expanded silica structure or a continuous random network) as well as the constraints imposed during the RMC procedure itself. This poses problems when for example trying to take the presence of defects into consideration, or when attempting to distinguish between amorphous phases of the same composition, formed by different methods. 26

Molecular simulations can also complement this experimental characterization, by providing a more direct look at the microscopic structure — and other physical and chemical properties — of the amorphous MOFs. However, most of the computational work published to date relies on a classical description of the interactions in the system, describing intra- and intermolecular interactions with parametrized force fields. These classical approaches cannot describe the breaking and formation of bonds, and thus cannot accurately describe the formation of disordered and amorphous phases of MOFs. Semi-classical approaches have also been employed, using so-called "reactive force fields", $27|28|$ but they are severely limited in their description of chemical events, having low accuracy. Moreover, they can only be used for certain classes of bonds (for which they have been parametrized).

In order to describe accurately the behavior of the systems undergoing chemical changes, it is necessary to use computationally expensive first principles methods, such as ab initio molecular dynamics. We have recently used it, in combination with in situ variable temperature X-ray and ex situ neutron pair distribution function experiments, to model the melting of ZIF materials and their liquid phase. $\frac{142930}{30}$ Moreover, it has been used extensively in the field of geophysics to study the properties of melts and glasses, ${ }^{31 / 32}$ including silicates ${ }^{33 / 34}$ which have some structural characteristics similar to ZIFs. The application of these existing techniques to MOF structures is however difficult given the more complicated nature of MOF structures.

In this work, we describe a study of the glass phases obtained by quenching liquid ZIFs through first-principles molecular dynamics simulations. It is the first direct computational description of MOF glasses, at the quantum chemical level. By computational modeling the melt-quench process on three different ZIF crystals, we aim at understanding the roles of the topology and the chemistry 
on the structure of the quenched phase as compared to the crystal and liquid. We detail both properties of the local order, such as interatomic distances and coordination angles, as well as probing remnant framework connectivity and porosity of the glass materials.

\section{Results and discussion}

In order to understand the nature of ZIF glasses from first-principles methods, without biasing the structures obtained through hypotheses made during their construction, our simulations followed a virtual "melt quench" sequence, entirely using first-principles DFT-based methods. In previous works, we showed how first-principles molecular dynamics (FPMD) could provide a description of the melting of ZIFs, and the properties of the liquid ZIFs obtained in this way. ${ }^{14[29}$ Here we followed a similar methodology, starting from the crystalline structures of three ZIF materials (ZIF-4, ZIF-8 and SALEM-2). SALEM-2 ${ }^{35}$ and ZIF-8 ${ }^{36}$ have the same sodalite (sod) topology, but different organic linker (imidazolate vs. 2-methylimidazolate, respectively), while ZIF-4 ${ }^{36}$ has cag topology. We first heated the crystalline structures to obtain a dynamics of the corresponding liquid. Then, ten different configurations from each MD simulation of a ZIF liquid were taken, and used as starting points for a rapid quenching — all details can be found in the Methods section and generate a molecular dynamics trajectory of each ZIF glass.

Because of the high computational cost of FPMD on systems with primitive cells ranging from 204 to 276 atoms (and dimensions up to $\sim 18 \AA$ ), we could not perform these simulations on supercells — which would reduce the impact of finite size effects and periodicity. Therefore, to obtain a good statistical description of the real glass configurations, which are disordered in nature, we performed multiple quenching simulations, and obtained 10 configurations for each of the 3 glasses studied. We then averaged the properties measured on all 10 configurations. Similar procedures, going to very high temperatures followed by a fast quenching, have been validated on other systems such as silica glasses before, with good results. 37 


\section{Local order: interatomic distances and bond angles}

To characterize the evolution of the coordination and framework associated with the quenching process, we first examined the total radial distribution function (RDF, see Figure S1). We see that on average, the glass phase obtained is structurally quite similar to the crystalline phase, especially as the local order (i.e. distances below $\sim 6 \AA$ ) dominates — highlighting how it can be difficult to obtain information on the detailed microscopic structure of such glasses through total scattering experiments. Even then, ZIF-8 seems to exhibit a greater difference compared to its crystalline form than the two others. We also observe that the variations between the ten different glass replicas are large, highlighting the disordered nature of the glass phase through variations of the local order. To make this visible, we will plot the glass average as a black solid line, and show the standard deviation as a light grey area (see Figure S1).

To look in more detail at the structure of the zinc-imidazolate three-dimensional networks in the glasses, we show in Figure 1 the radial distribution functions for the $\mathrm{Zn}-\mathrm{Zn}$ atom pairs, and in Figure S2 for the $\mathrm{Zn}-\mathrm{N}$ pairs. We can see that in both cases the thermal widening of the first peak, induced by melting, vanishes as the structures are cooled down. Looking more closely at the differences between the frameworks, we see that the substitution of the imidazolate in ZIF-8 impacts its behaviour upon quenching, compared to SALEM-2. For instance, in the Zn-N RDF (Figure S2) we clearly see that no nitrogen atoms are being trapped in the 2.5-3.8 $\AA$ region for ZIF-8, while it is the case in ZIF-4 and SALEM-2. This is probably due to the better stability of the "perpendicular" state (when the plane of the imidazolate is turned $90^{\circ}$ from its original position) for the non-substituted imidazolate - this is consistent with results obtained from previous computational studies of the melting of ZIF-4 and ZIF-8. $\underline{29}$

This has further consequences on the distances between the edges of the coordination network, namely the zinc cations, as evidenced by the cumulative curves shown in Figure 1. Indeed, as shown in Table 1, the distances with the fourth closest neighboring zinc atom are differently affected by melting and quenching. The melting increases this distance by more than $15 \%$ in both

ZIF-8 and SALEM-2 and only $1.5 \%$ in ZIF-4, confirming our previous findings. $\frac{29}{2}$ Similarly for 

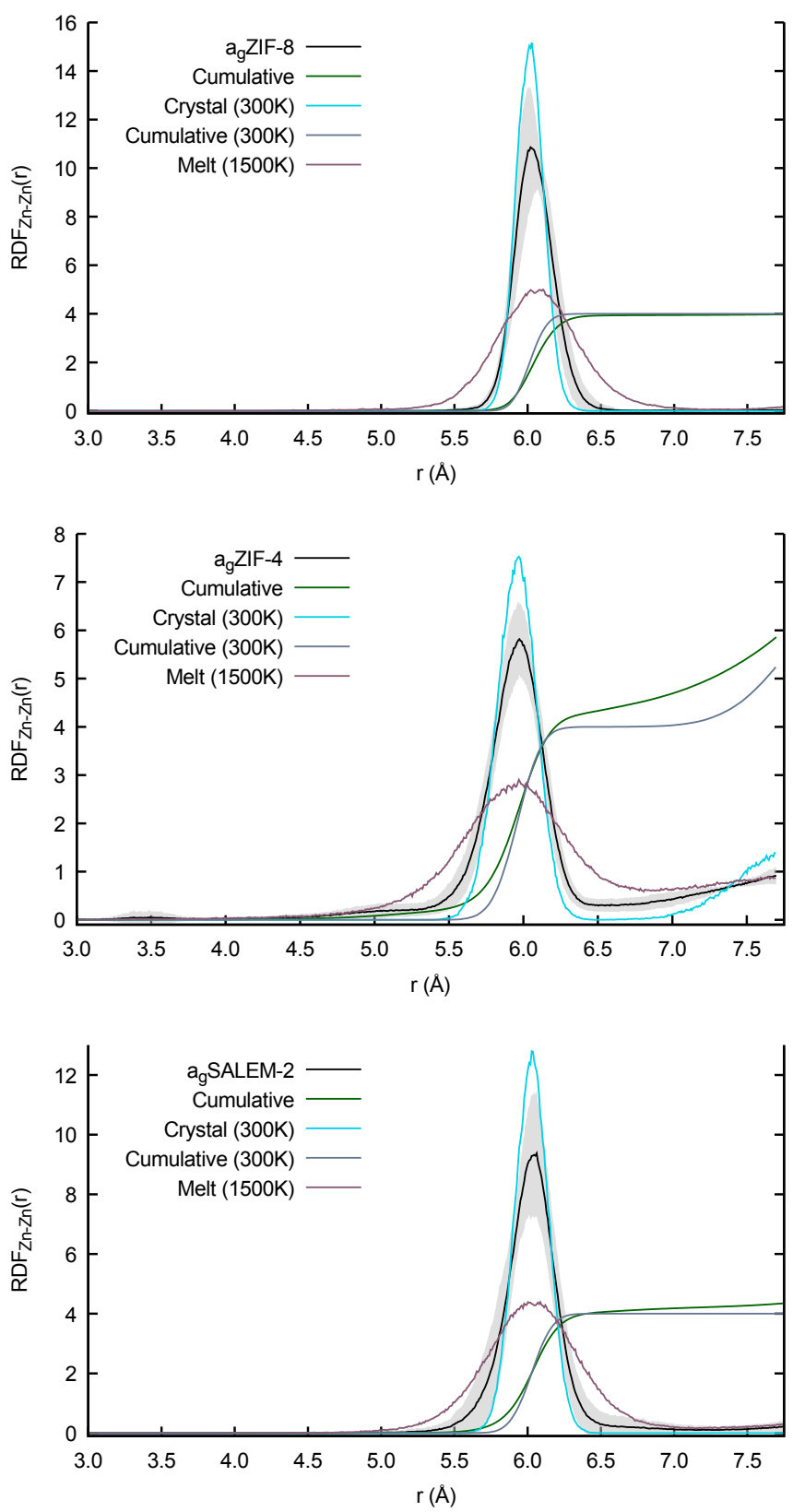

Figure 1: Radial distribution functions (RDF) for the $\mathrm{Zn}-\mathrm{Zn}$ atom pairs. Light blue: crystalline RDF at $300 \mathrm{~K}$; purple: average RDF for the melt at 1,500 K; black: average RDF over all glass configurations; The light gray areas correspond to the standard deviation between the ten glass replicas (see text for details). Cumulative functions corresponding to the number of neighbors are shown in green for the glass, and in blue for the crystal. 
Table 1: Average distances to the third and fourth neighboring zinc atoms from a zinc for the three frameworks at different temperatures and in the glass phase.

\begin{tabular}{cccc}
\cline { 2 - 4 } & ZIF-8 $\left(3^{\text {rd }}-4^{\text {th }}\right)$ & ZIF-4 $\left(3^{\text {rd }}-4^{\text {th }}\right)$ & SALEM-2 $\left(3^{\text {rd }}-4^{\text {th }}\right)$ \\
\hline Crystal $(300 \mathrm{~K})$ & $6.1-6.5 \AA$ & $6.1-6.5 \AA$ & $6.1-6.5 \AA$ \\
Melt $(1500 \mathrm{~K})$ & $6.3-7.6 \AA$ & $6.2-6.6 \AA$ & $6.3-7.5 \AA$ \\
Glass $(300 \mathrm{~K})$ & $6.1-8.0 \AA$ & $6.1-6.2 \AA$ & $6.1-6.4 \AA$ \\
\hline
\end{tabular}

Table 2: Average value of the tetrahedral angle and its standard deviation for the three frameworks in the crystal, the melt and the glass. For the glass phase, the standard deviation indicated after \pm is the average of the standard deviations observed for the ten replicas, obtained from independent initial configurations.

\begin{tabular}{cccc}
\cline { 2 - 4 } & ZIF-8 & ZIF-4 & SALEM-2 \\
\hline Crystal $(300 \mathrm{~K})$ & $111.3^{\circ}( \pm 1.4)$ & $112.2^{\circ}( \pm 1.6)$ & $111.6^{\circ}( \pm 1.6)$ \\
Melt $(1500 \mathrm{~K})$ & $115.2^{\circ}( \pm 4.0)$ & $117.3^{\circ}( \pm 3.7)$ & $116.0^{\circ}( \pm 4.1)$ \\
Glass $(300 \mathrm{~K})$ & $112.5^{\circ}( \pm 2.4)$ & $113.3^{\circ}( \pm 2.2)$ & $112.7^{\circ}( \pm 2.4)$ \\
\hline
\end{tabular}

the quenching process, the chemistry has an important impact. This distance actually comes back to lower values than the one in the crystalline phases for ZIF-4 and SALEM-2 — while increasing, to even higher values than in the liquid, in ZIF-8. We therefore see that the loss of order during melting is mainly influenced by the topology, but that the precise chemical interactions play a larger role in the partial reconstruction of the coordination network during quenching to obtain a glass phase.

As a measure of local order around the zinc atoms, we investigated the $\mathrm{N}-\mathrm{Zn}-\mathrm{N}$ angle, or tetrahedral angle, which can measure the distortion in the $\mathrm{Zn}^{2+}$ coordination environment. ${ }^{38}$ Figure S3 presents the distributions of $\mathrm{N}-\mathrm{Zn}-\mathrm{N}$ angle for the three frameworks in the crystalline phase (at $300 \mathrm{~K}$ ), in the melt (at 1,500 K) and in the glass phase (at $300 \mathrm{~K}$ ). The distributions are all quasiGaussian, although stretched to higher angles marking the asymmetry of the bending potential (due to steric repulsion at low angles). The thermal widening of the distributions in the melt is clearly visible, and we note with interest that this disorder is partially conserved in the glass phase. In the melt, the average angle also increases, mainly due to the undercoordination of the zinc atoms: more 
available space and lower steric constraints lead to a larger "spread" between the linkers that are present. Table 2 summarizes the main features of these angle distributions, i.e. the average values and associated standard deviations. In liquid ZIF-4, the presence of $60 \%$ of four-fold coordinated zinc ions is associated with an increase of $4.5 \%$ of the average tetrahedral angle, whereas the $74 \%$ of four-fold coordination in SALEM-2 leads to an increase of $3.9 \%$, and the $81 \%$ in ZIF-8 to an increase of $3.5 \%$.

Despite these differences upon melting, the average $\mathrm{N}-\mathrm{Zn}-\mathrm{N}$ angle values obtained in the glass phase present surprisingly small deviations from the crystal value. This is an indication of the important reorganization that occurs during quenching, even at very high quenching rate (as is the case in our MD protocol). ZIF-4 has, by a small amount, the smallest deviation of $0.98 \%$ of the average tetrahedral angle (compared to $0.99 \%$ and $1.1 \%$ for SALEM-2 and ZIF-8 respectively). This indicates a greater reconstruction of the coordination network in ZIF-4 compared to ZIF8 and SALEM-2, with a decrease corresponding to $3.5 \%$ of the crystalline value (vs. $2.9 \%$ and $2.4 \%$ for SALEM-2 and ZIF-8 respectively). This is also attested by the standard deviation of the distributions: it is $71 \%, 50 \%$, and $38 \%$ higher in the ZIF-8, SALEM-2 and ZIF-4 glasses as compared to their crystalline counterparts.

\section{Topological changes of the coordination network}

In order to have a global view on the evolution of the coordination network in the formation of the ZIF glasses, we first calculated the $\mathrm{Zn}-\mathrm{N}$ coordination numbers. Figure 2 shows the distributions of coordination numbers (discrete values, calculated with a threshold at $2.5 \AA$ ) of nitrogen atoms around each zinc atom. The strong increase of four-fold coordination, from the undercoordinated liquid to the glass, is a direct confirmation at the microscopic level of the reconstruction mechanism, as already suggested in $\underline{11}$ for ZIF-4. The proportion of four-fold coordinated zincs in ZIF-4 goes from $60 \%$ to $93 \%$ through quenching, regaining an average coordination number of 3.93 (compared to the crystalline value of 4.0). This $33 \%$ increase in ZIF-4 is larger than in SALEM-2 $(22 \%)$ and ZIF-8 (17\%) undergoing the same thermal process. 


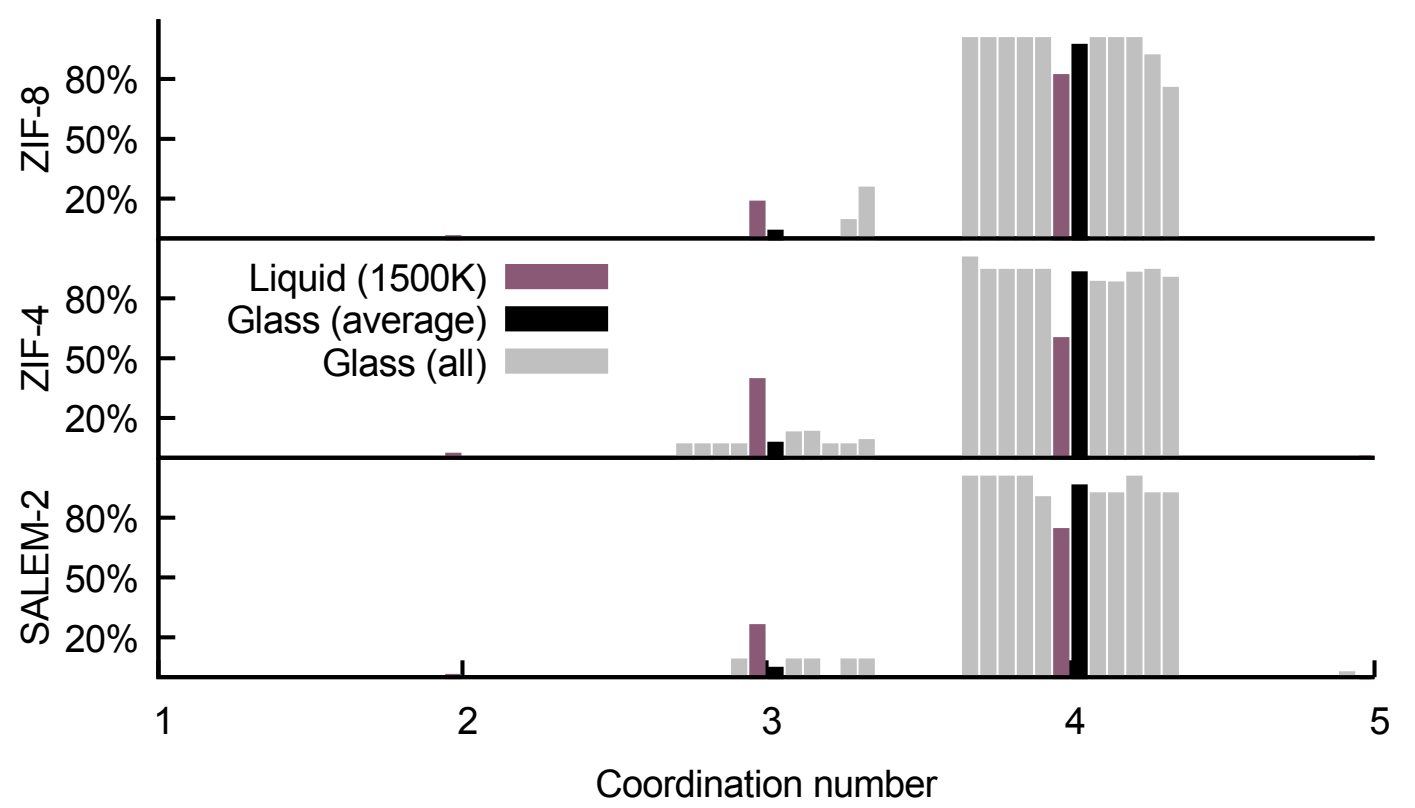

Figure 2: Distribution of the zinc-nitrogen coordination number (discrete values). The threshold is set here at $2.5 \AA$ used, as determined from the crystal and melt simulations, see details in the text.

To unravel the role of the topology, we carried out analyses of the statistics of $\mathrm{Zn}$-Im ring sizes in our systems. Analysis of this type is common for microporous materials, and has been used to characterize amorphous $\mathrm{GeS}_{2}$ and $\mathrm{SiO}_{2}$ systems. ${ }^{39}$ Figure 3 shows the distributions of ring sizes for the three frameworks in the liquid phase at different temperatures, and in the melt-quenched glasses. On this figure, the relative stabilization of ZIF-4 during melting and quenching compared to ZIF-8 or SALEM-2 appears clearly. Indeed we see that upon heating and melting, the number of 8-member rings decreases by a quarter at 1,500 K in ZIF-4, upon which there is formation of other ring structures with size 4, 6, and 10. On the contrary, in ZIF-8 and SALEM-2, the loss of 8member rings does not coincide with the formation of any other structures in significant amounts. During quenching, the number of 8-member rings grows back for ZIF-4, while it dramatically decreases for ZIF-8 and SALEM-2. Therefore, this analysis confirms that the local topology is at the same time less affected in ZIF-4 during melting and better recovered during quenching compared to ZIF-8 and SALEM-2. We also see, again, that there are large variations between the individual glass configurations, demonstrating the important disorder in that state.

These structural observations demonstrate the importance of the ZIF materials' topology, rather 

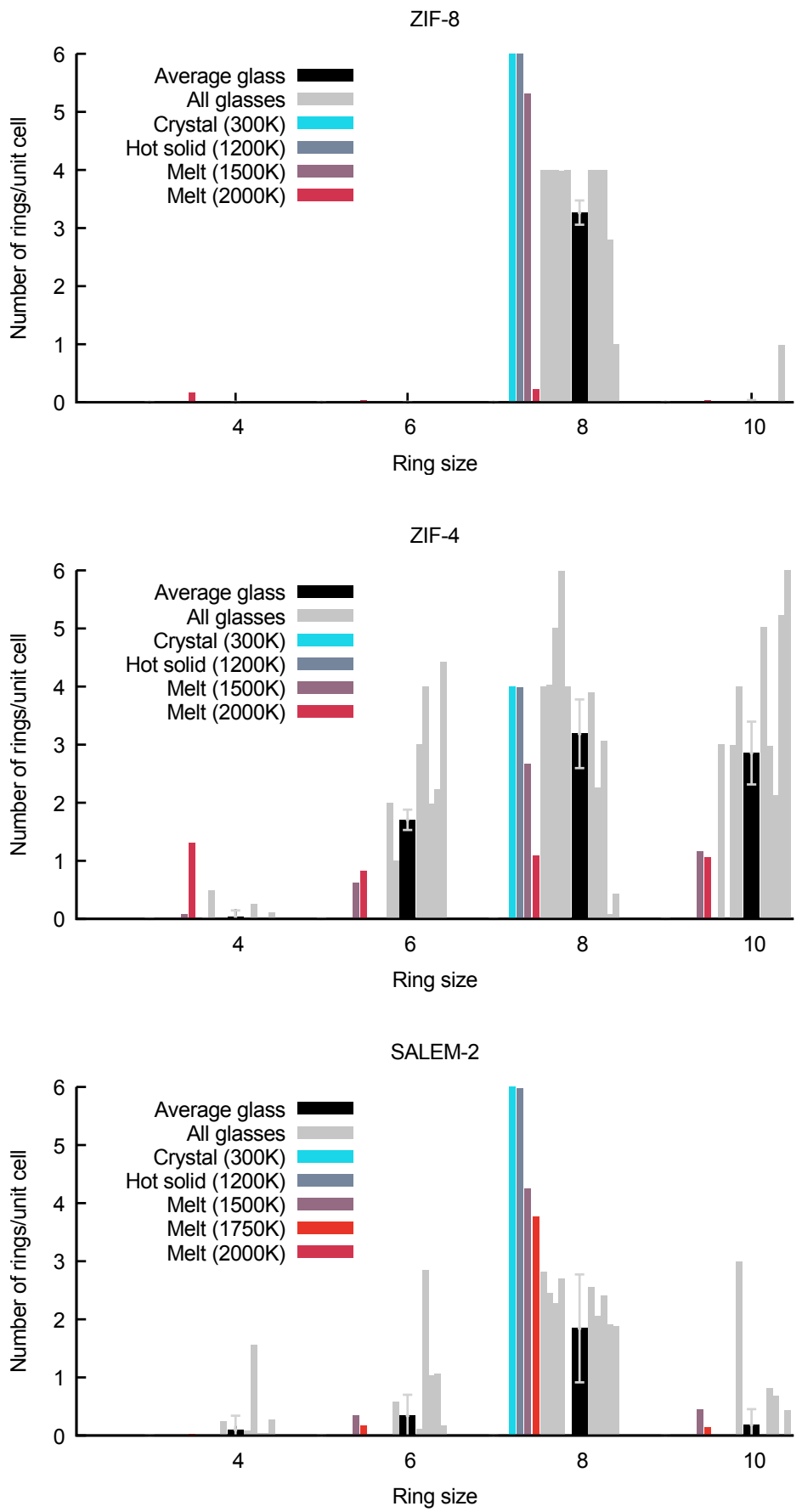

Figure 3: Distributions of the sizes of zinc-imidazolate alternate rings for the three frameworks in their crystalline (light blue), liquid (purple, red and dark red) and glass (black) phases. The light gray bars correspond to the ten glass configurations for each framework. 
than their chemistry, as an influence on the quenching behaviour: SALEM-2 and ZIF-8 have the same sod topology, and exhibit more similarity than ZIF-4 (which has cag topology). Indeed, except in the case of the zinc-zinc distances, where the substitution of the imidazolate seems to play a larger role, all the properties linked to the structure of the coordination network point to the larger role of the initial topology in the quenching behaviour.

\section{Thermodynamics of the glass phase}

To characterize the stability of the nature of the $\mathrm{Zn}-\mathrm{N}$ bond in the glass, and compare it to that known in the crystal and melt, we calculated the potential of mean force (PMF), or free energy profile, along the $\mathrm{Zn}-\mathrm{N}$ coordinate. From the partial radial distribution function between $\mathrm{Zn}$ and $\mathrm{N}, g_{\mathrm{Zn}-\mathrm{N}}(r)$, the PMF is defined as $F(r)=-k_{\mathrm{B}} T \ln g(r)$, where $k_{\mathrm{B}}$ is the Boltzmann constant. Figure 4 presents these potentials of mean force in unit of $k_{\mathrm{B}} T$ - allowing for a better comparison between temperatures.

We first note that no free energy barrier can be extracted for ZIF-8-based glass, as there are no nitrogen at a distance between 2.6 and $3.8 \AA$ during the time of our simulations. On the contrary, for ZIF-4 and SALEM-2 glasses, free energy barriers can be extracted, even though no ligand exchange events are observed during the simulation. These barriers are small in absolute value, with 22 and $27 \mathrm{~kJ} / \mathrm{mol}$ for ZIF-4 and SALEM-2 respectively. However, at $300 \mathrm{~K}$ these barriers represent a height of about $10 k T$ and therefore their crossing will be a very rare event. We can compare these values with those of the liquid ZIFs, for which we have determined the free energy barriers as a function of temperature: see Ref. 29 for the thermodynamic properties of the ZIF-4 liquid; for SALEM-2 we found that $\Delta U_{\mathrm{Zn}-\mathrm{N}}^{\ddagger}=177 \mathrm{~kJ} / \mathrm{mol}$ and $\Delta S_{\mathrm{Zn}-\mathrm{N}}^{\ddagger}=59 \mathrm{~J} \cdot \mathrm{mol}^{-1} \cdot \mathrm{K}^{-1}$. Therefore, the temperatures at which the barriers in the liquid have the same value (in unit of $k T$ ) are $1135 \mathrm{~K}$ and $1176 \mathrm{~K}$ for ZIF-4 and SALEM-2 respectively. Therefore, from the perspective of the Zn-N bond strength, these glass phases thus appears to be like "frozen" liquid just below the melting temperature.

As already mentioned in the examination of the $\mathrm{Zn}-\mathrm{N}$ radial distribution function, the precise 

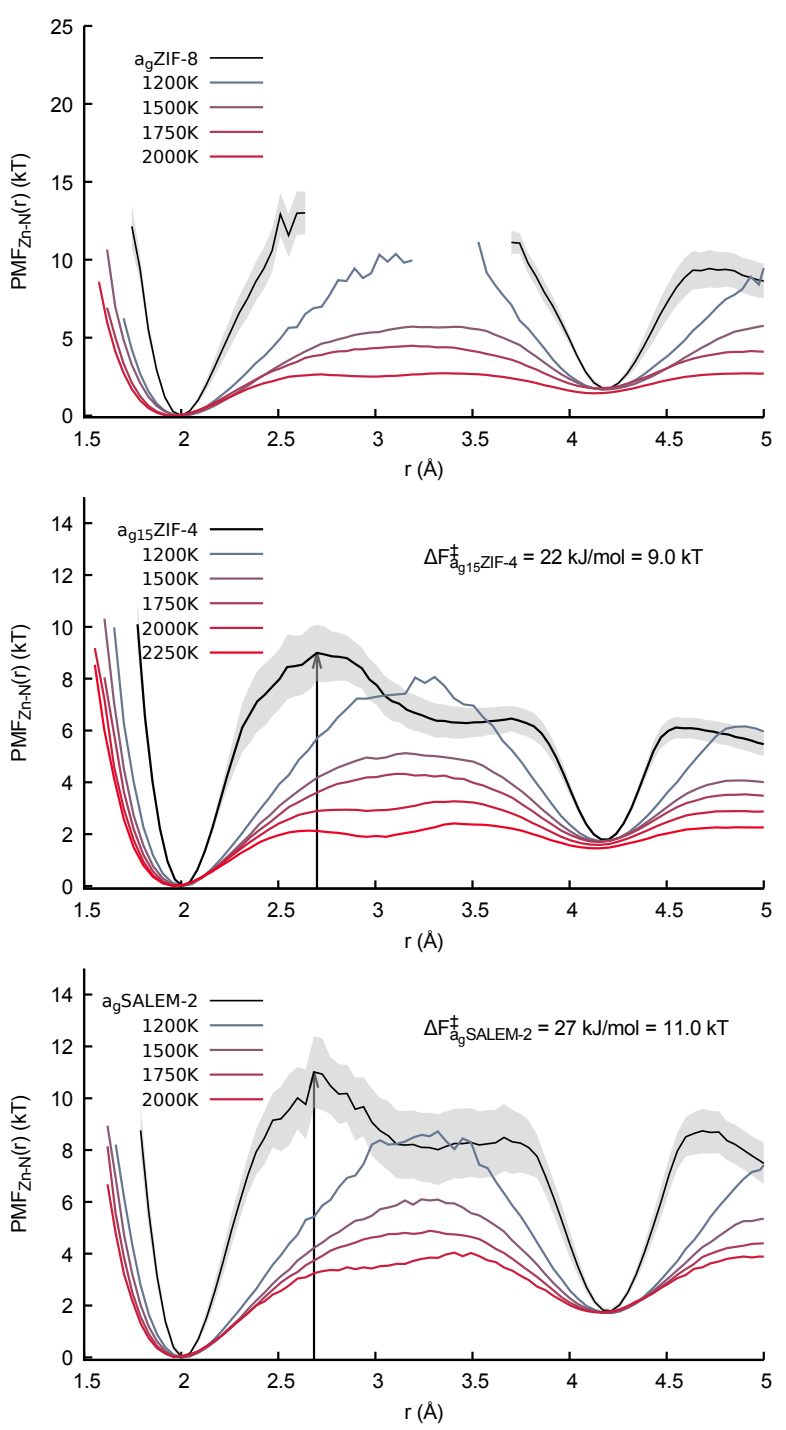

Figure 4: Potentials of mean force (PMF) along the $\mathrm{Zn}-\mathrm{N}$ coordinate for the three structures in unit of $k \mathrm{~T}$. In red colors the evolution with the elevation of temperature are reminded and in black the average PMF over all the glass configurations. As previously described the light gray areas correspond to the standard deviation between the ten trajectories. 
chemical interaction between the ligand and the zinc seems to be a major factor in the thermodynamics of the bond as part of the coordination network. Indeed, while ZIF-4 and SALEM-2 conserve configurations where an imidazolate ring is perpendicular to a zinc (in the sense that the normal vector to the plan of the imidazolate goes towards the zinc) and thus exhibit $\mathrm{Zn}-\mathrm{N}$ distances between 2.6 and $3.8 \AA$, ZIF-8 seems to rearrange enough to avoid this behaviour.

\section{Porosity}

Finally, we used computational tools to characterize the porosity left in the glasses after quenching — in particular, we compare it to the porosity in the liquid melts, a surprising feature revealed in our previous studies. ${ }^{14}$ Both MOF liquids and MOF glasses can present microporosity, with typical pore sizes in MOF glasses much smaller than those found in mesoporous and macroporous glasses obtained by, e.g., spinodal decomposition. This characterization is based on the instantaneous geometries extracted at regular points from our FPMD trajectories.

Figure 5 presents the distributions of total porous volumes for the three frameworks in the crystalline phase, in the melt and in the glass phase. These graphs reinforce the idea that the topology governs the disorder impacted on the crystalline framework upon heating, and the partial reconstruction of the framework upon cooling. Indeed, although the average porous volumes of ZIF-8 and SALEM-2 in the glass are 249 and $377 \mathrm{~cm}^{3} / \mathrm{kg}$ respectively compared to a smaller value of $67 \mathrm{~cm}^{3} / \mathrm{kg}$ for the ZIF-4 glass, the latter sees a much larger increase, relative to the crystalline value, during the formation of the glass. The average porous volume is $29 \%$ higher for ZIF-4 than it is in the crystalline phase, whereas for ZIF-8 it is 3.5\% lower and almost unchanged (1\% higher) in SALEM-2. Moreover, the average values observed in the ZIF-8 and SALEM-2 glasses, although close to their crystalline equivalent, have to be tempered by the large proportion of configurations with no porosity at all, accounting for about $20 \%$ of configurations in ZIF-8 and 30\% in SALEM-2.

When we start looking not at total porous volume, but at the average accessible volume - i.e., the pore volume excluding nonconnected void pockets —, the difference is even more pronounced between ZIF-4 and the other two frameworks (Table 3). For ZIF-8 and SALEM-2, the accessible 

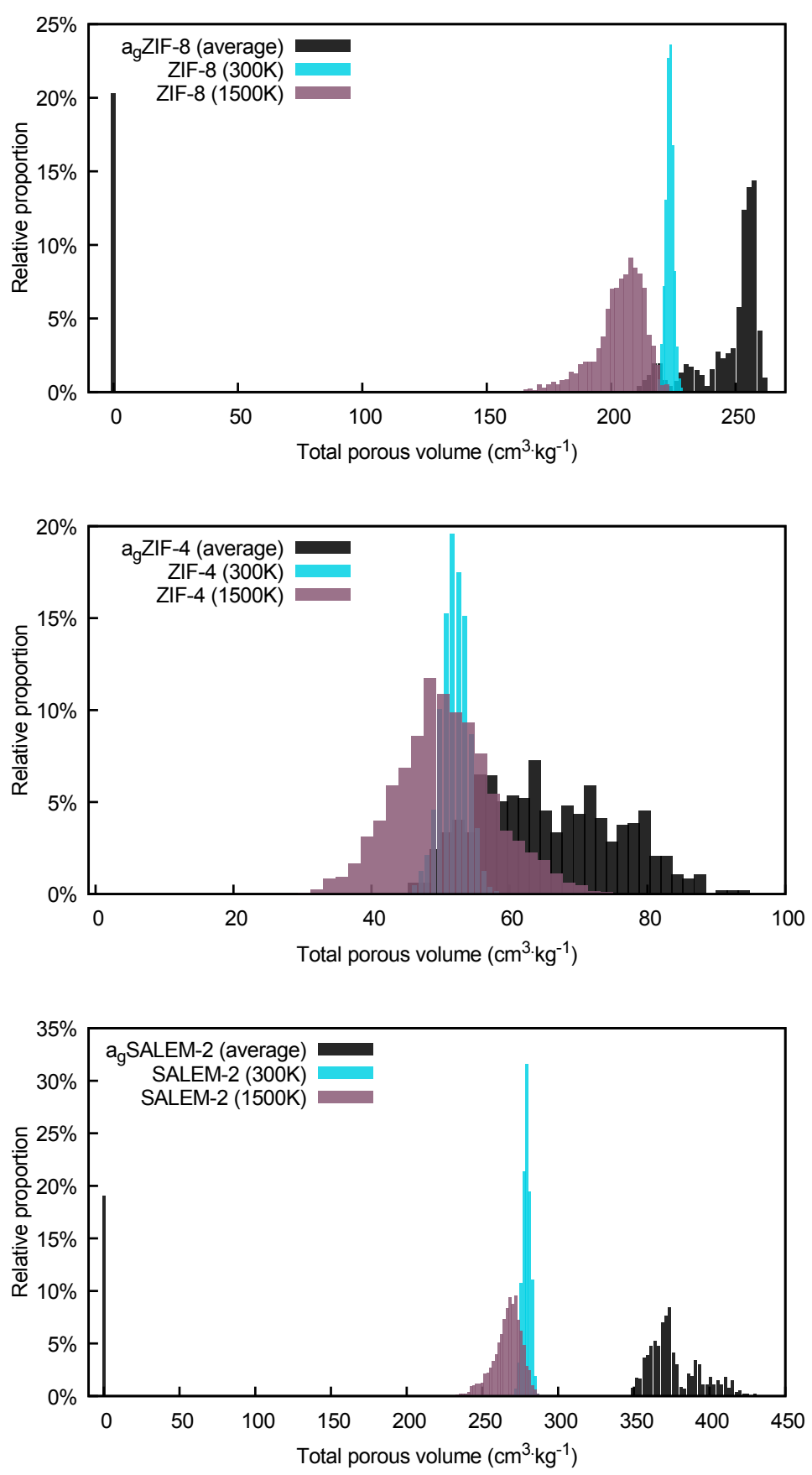

Figure 5: Total porous volumes for the three frameworks in all phases: crystalline (light blue), melt (purple) and glass (black). The light gray curves correspond to the ten different initial configurations. 
volume and the total porous volume coincide for a helium probe, so the decrease in ZIF-8 and the small increase in SALEM-2 are unchanged. However, for ZIF-4, the melting process seems to open larger channels than in the crystal, leading to an increase in accessible volume of $64 \%$. Actually, in the crystal the average accessible volume was only $74 \%$ of the total porous volume, while it represents $96 \%$ in the glass. This result is in line with the experimental findings on the porosity of the ZIF-4 glass analyzed by positron annihilation lifetime spectroscopy (PALS) ${ }^{40}$ We note that the large magnitude of the increase we observe here is probably exacerbated by the fact that we do not take into account the possible densification as ZIF-4 is molten and quenched. Nonetheless, the opening of larger voids is confirmed by the PALS analyses which provides another validation of the methods we used.

\section{Conclusions and perspectives}

We studied the quenching of ZIF-4, ZIF-8 and SALEM-2 liquids by ab initio molecular dynamics simulations, in order to characterize the resulting glass configurations and compare their properties to both the crystalline and liquid phases of the same compound. It is important to note that this computational setup is not a totally realistic process, for multiple reasons. First, because the quenching rate attainable in MD simulations is much faster than experimentally; secondly, because these ZIFs may not necessarily melt experimentally, due to their melting point being above the decomposition temperature of the linker. ${ }^{14}$ However, despite these limitations, we can draw some interesting conclusions from these simulations, which are the first description of MOF glasses at the quantum chemical level, i.e. with at a level of theory that allows a chemically accurate description of the coordination bonds breaking and forming.

Table 3: Average accessible volumes for the three frameworks in the crystalline and the glass phase.

\begin{tabular}{cccc}
\cline { 2 - 4 } & ZIF-8 & ZIF-4 & SALEM-2 \\
\hline Crystal & $258 \mathrm{~cm}^{3} / \mathrm{kg}$ & $39 \mathrm{~cm}^{3} / \mathrm{kg}$ & $372 \mathrm{~cm}^{3} / \mathrm{kg}$ \\
Glass & $249 \mathrm{~cm}^{3} / \mathrm{kg}$ & $64 \mathrm{~cm}^{3} / \mathrm{kg}$ & $377 \mathrm{~cm}^{3} / \mathrm{kg}$ \\
\hline
\end{tabular}


The comparison between the frameworks indicates that the substitution of the imidazolate linker and the topology of the frameworks both have roles in the behaviour upon quenching, and the properties of the glasses obtained. However, the topology of ZIF-4, allowing rearrangement of the ring structures during melting and partial recovery during quenching, seems to play a large role in the stabilization of this framework with regard to melting and quenching. In fact, SALEM-2, though it has the same unsubstituted imidazolate ligand, sees a collapse of its topology which, far from being countered by quenching, seems to be reinforced by it. On the other hand, when looking only at the local distances between the nodes of the framework, the zinc cations, SALEM-2 behaves more like ZIF-4 than ZIF-8 in the sense that quenching makes it look more like the crystal. The proportion of four-fold coordinated zinc ions also increases more (upon quenching) in SALEM-2 than in ZIF-8, but still much less than in ZIF-4.

These results open many interesting questions on the field of MOF-glasses, which might only be addressed through combined experimental and computational approaches. Particularly, the influence of the original crystalline framework upon glass structure and porosity will be of great importance given efforts to develop MOF-glass membranes for gas separations. ${ }^{41}$ Equally, information on the tunability of glass structures through accurate controlling of quenching speed, or consideration of the evolution of liquid structure upon continued heating, would be extremely valuable in tailoring MOF-glass properties. A promising theoretical development would be to directly simulate with classical molecular mechanics methods the glass structures obtained by reverse Monte-Carlo (RMC) modeling, and compare such results with the ab initio structures obtained herein. This would allow to get information on the structure and dynamics of the glass at larger scale in order to have a better view of the statistical disorder and the thermodynamics of these new exciting materials - while at the same time validating the microscopic details of these RMC structures against quantum chemical structures

Equally, the simulation of the melting and quenching processes with classical methods relying on reactive force-fields, where coordination bonds can be broken, still requires further force-field development, but their development would facilitate access to large length and time scales, and at 
the same time provide further information on the presence of coordinatively unsaturated metal sites in the final glass structure, which would point towards applications in catalysis. Finally, less computationally expensive methods shall be used to screen many more frameworks and draw generic criteria for the capability of melting and the glass-forming ability based on precise characteristics of the topology and the chemistry (connectivity, openness, isolated interaction strength). The ab initio data collected during our work may be used as a training data set for the optimization of future classical or semi-classical force fields. 


\section{Methods}

\section{First principles molecular dynamics}

The behaviour of zeolitic imidazolate frameworks as a function of temperature was studied by means of density functional theory (DFT)-based molecular dynamics (MD) simulations, using the Quickstep module ${ }^{42}$ of the CP2K software package. ${ }^{43}$ We used the hybrid Gaussian and plane wave method GPW ${ }^{44}$ as implemented in $\mathrm{CP} 2 \mathrm{~K}$. The simulations were performed in the constantvolume $(N, V, T)$ ensemble with fixed size and shape of the unit cell. A timestep of $0.5 \mathrm{fs}$ was used in the MD runs, the temperature was controlled by velocity rescaling. ${ }_{45}$

We used parameters already fine-tuned for similar systems in previous works. $\frac{14 \mid 29}{}$ In particular, the exchange-correlation energy was evaluated in the PBE approximation, $\frac{46}{}$ and the dispersion interactions were treated at the DFT-D3 level. ${ }^{47}$ The Quickstep module uses a multi-grid system to map the basis functions onto, with 4 different grids, a plane-wave cutoff for the electronic density of $600 \mathrm{Ry}$, as already used in Ref. 48, and a relative cutoff of $40 \mathrm{Ry}$. Valence electrons were described by double- $\zeta$ valence polarized basis sets and norm-conserving Goedecker-Teter-Hutter 49 pseudopotentials all adapted for PBE (DZVP-GTH-PBE) for H, C and $\mathrm{N}$ or optimized for solids (DZVP-MOLOPT-SR-GTH) in the case of Zn.

The simulation box chosen for ZIF-4 (space group $\mathrm{Pbca}$ ) is the orthorhombic primitive unit cell, containing 272 atoms, with cell parameters $a=15.423 \AA$, $b=15.404 \AA, c=18.438 \AA$, and $\alpha=\beta=\gamma=90^{\circ}$. The simulation box chosen for ZIF-8 (space group $I \overline{4} 3 m$ ) is the orthorhombic primitive unit cell, containing 276 atoms, with cell parameters $a=b=c=16.993 \AA$, and $\alpha=$ $\beta=\gamma=90^{\circ}$. The simulation box chosen for SALEM-2 (space group $I \overline{4} 3 m$ ) is the orthorhombic primitive unit cell, containing 204 atoms, with cell parameters $a=b=c=17.014$ Aand $\alpha=\beta=$ $\gamma=90^{\circ}$. Representative input files for the molecular dynamics simulations are available online in our data repository at https://github.com/fxcoudert/citable-data.

For all structures (ZIF-4, ZIF-8 and SALEM-2), we conducted simulations at 1,500 K starting from the crystalline structure. Then, for ZIF-4 we took ten configurations of the liquid phase corre- 
sponding to snapshots of the equilibrated trajectory after $6 \mathrm{ps}$ (11 ps after the beginning with $5 \mathrm{ps}$ of equilibration) and every $15 \mathrm{ps}$ after that up to $141 \mathrm{ps}$. We took the exact same snapshot for ZIF-8 on the trajectory at 1,500 K. For SALEM-2, the first configuration is the snapshot after $55 \mathrm{ps}$ of the equilibrated trajectory and the last one is the one after $190 \mathrm{ps}$. The choice of $1,500 \mathrm{~K}$ was made as it was the lowest temperature at which the three frameworks exhibited liquid-like behaviour, either by looking at free diffusion or cleavage frequencies of $\mathrm{Zn}-\mathrm{N}$ bonds.

From these $30(=10 \times 3)$ configurations, we launched consecutive 4 ps constant temperature simulations at $1,300 \mathrm{~K}, 1,100 \mathrm{~K}, 900 \mathrm{~K}, 700 \mathrm{~K}, 500 \mathrm{~K}$, and finally $300 \mathrm{~K}$. The instantaneous temperature followed a well-defined ramp as the time constant we chose for the thermostat was of 1 ps before reaching room temperature $(300 \mathrm{~K})$. We restarted the simulations at $300 \mathrm{~K}$ to reach at least $70 \mathrm{ps}$ in total. All properties of interest were then averaged over the part of the trajectory after $25 \mathrm{ps}$ and up to at least $70 \mathrm{ps}$ (over $45 \mathrm{ps}$ minimum).

\section{Trajectory analysis}

The coordination number for nitrogen atoms around the zinc cation is computed by taking a cut-off radius of $2.5 \AA$, a value chosen from the $\mathrm{Zn}-\mathrm{N}$ partial radial distribution function at room temperature. We checked that the precise value used does not influence the outcome of the calculations, nor does the choice of a discontinuous criterion (compared to the use of a damping function near the cut-off value).

In order to compute the total porous volume, we used the Zeo++ software. ${ }^{\sqrt[50-52]{-51}}$ It relies on a geometric decomposition of space to compute the accessible and non-accessible volume to a sphere of a given radius. We have taken a value a $1.2 \AA$ simulating the porous volume as seen by a helium molecule, calculating the distribution of instantaneous total pore space (sum of the accessible and the non-accessible volume) along the MD trajectories. 


\section{Supporting Information}

Total radial distribution function, mean square displacements, porous volumes, crystalline structures. Additional statistics on the mechanism of bond cleavage and diffusion coefficients.

\section{Acknowledgements}

We thank Anne Boutin and Alain Fuchs for fruitful discussions. This work benefited from the financial support of ANRT (thèse CIFRE 2015/0268) and access to HPC platforms provided by a GENCI grant (A0070807069).

\section{References}

(1) Kinoshita, Y.; Matsubara, I.; Higuchi, T.; Saito, Y. The Crystal Structure of Bis(adiponitrilo)copper(I) Nitrate. Bull. Chem. Soc. Jpn. 1959, 32, 1221-1226.

(2) Hoskins, B. F.; Robson, R. Infinite polymeric frameworks consisting of three dimensionally linked rod-like segments. J. Am. Chem. Soc. 1989, 111, 5962-5964.

(3) Li, H.; Eddaoudi, M.; O'Keeffe, M.; Yaghi, O. M. Design and synthesis of an exceptionally stable and highly porous metal-organic framework. Nature 1999, 402, 276-279.

(4) Zhou, H.-C.; Long, J. R.; Yaghi, O. M. Introduction to Metal-Organic Frameworks. Chem. Rev. 2011, 112, 673-674.

(5) Furukawa, H.; Cordova, K. E.; O'Keeffe, M.; Yaghi, O. M. The chemistry and applications of metal-organic frameworks. Science 2013, 341, 1230444-1230444.

(6) Doonan, C. J.; Sumby, C. J. Metal-organic framework catalysis. CrystEngComm 2017, 19, 4044-4048. 
(7) Sumida, K.; Rogow, D. L.; Mason, J. A.; McDonald, T. M.; Bloch, E. D.; Herm, Z. R.; Bae, T.-H.; Long, J. R. Carbon Dioxide Capture in Metal-Organic Frameworks. Chem. Rev. 2011, 112, 724-781.

(8) Liu, Y.; Howarth, A. J.; Vermeulen, N. A.; Moon, S.-Y.; Hupp, J. T.; Farha, O. K. Catalytic degradation of chemical warfare agents and their simulants by metal-organic frameworks. Coord. Chem. Rev. 2017, 346, 101-111.

(9) Bennett, T. D.; Cheetham, A. K.; Fuchs, A. H.; Coudert, F.-X. Interplay between defects, disorder and flexibility in metal-organic frameworks. Nature Chem. 2017, 9, 11-16.

(10) Masoomi, M. Y.; Morsali, A.; Dhakshinamoorthy, A.; Garcia, H. Mixed-Metal MOFs: Unique Opportunities in Metal-Organic Framework (MOF) Functionality and Design. Angew. Chem. Int. Ed. 2019, 58, 15188-15205.

(11) Bennett, T. D.; Tan, J.-C.; Yue, Y.; Baxter, E.; Ducati, C.; Terrill, N. J.; Yeung, H. H. M.; Zhou, Z.; Chen, W.; Henke, S.; Cheetham, A. K.; Greaves, G. N. Hybrid glasses from strong and fragile metal-organic framework liquids. Nature Comm. 2015, 6, 8079.

(12) Lohe, M. R.; Rose, M.; Kaskel, S. Metal-organic framework (MOF) aerogels with high micro- and macroporosity. Chem. Commun. 2009, 6056.

(13) Bueken, B.; Van Velthoven, N.; Willhammar, T.; Stassin, T.; Stassen, I.; Keen, D. A.; Baron, G. V.; Denayer, J. F. M.; Ameloot, R.; Bals, S.; De Vos, D.; Bennett, T. D. Gelbased morphological design of zirconium metal-organic frameworks. Chem. Sci. 2017, 8, 3939-3948.

(14) Gaillac, R.; Pullumbi, P.; Beyer, K. A.; Chapman, K. W.; Keen, D. A.; Bennett, T. D.; Coudert, F.-X. Liquid metal-organic frameworks. Nature Mater. 2017, 16, 1149-1154.

(15) Tuffnell, J. M.; Ashling, C. W.; Hou, J.; Li, S.; Longley, L.; Ríos Gómez, M. L.; Bennett, T. D. 
Novel metal-organic framework materials: blends, liquids, glasses and crystal-glass composites. Chem. Commun. 2019, 55, 8705-8715.

(16) Bennett, T. D.; Horike, S. Liquid, glass and amorphous solid states of coordination polymers and metal-organic frameworks. Nat Rev Mater 2018, 3, 431-440.

(17) Orellana-Tavra, C.; Baxter, E. F.; Tian, T.; Bennett, T. D.; Slater, N. K. H.; Cheetham, A. K.; Fairen-Jimenez, D. Amorphous metal-organic frameworks for drug delivery. Chem. Commun. 2015, 51, 13878-13881.

(18) Chapman, K. W.; Sava, D. F.; Halder, G. J.; Chupas, P. J.; Nenoff, T. M. Trapping Guests within a Nanoporous Metal-Organic Framework through Pressure-Induced Amorphization. J. Am. Chem. Soc. 2011, 133, 18583-18585.

(19) Bennett, T. D.; Cheetham, A. K. Amorphous metal-organic frameworks. Acc. Chem. Res. 2014, 47, 1555-1562.

(20) Ghazy, A.; Safdar, M.; Lastusaari, M.; Karppinen, M. Amorphous-to-crystalline transition and photoluminescence switching in guest-absorbing metal-organic network thin films. Chem. Commun. 2020, 56, 241-244.

(21) Zhao, Y.; Lee, S.-Y.; Becknell, N.; Yaghi, O. M.; Angell, C. A. Nanoporous transparent MOF glasses with accessible internal surface. J. Am. Chem. Soc. 2016, 138, 10818-10821.

(22) Coudert, F.-X. Responsive metal-organic frameworks and framework materials: under pressure, taking the heat, in the spotlight, with friends. Chem. Mater. 2015, 27, 1905-1916.

(23) Bennett, T. D.; Saines, P. J.; Keen, D. A.; Tan, J.-C.; Cheetham, A. K. Ball-milling-induced amorphization of zeolitic imidazolate frameworks (ZIFs) for the irreversible trapping of iodine. Chem. Eur. J. 2013, 19, 7049-7055.

(24) Bennett, T. D.; Goodwin, A. L.; Dove, M. T.; Keen, D. A.; Tucker, M. G.; Barney, E. R.; 
Soper, A. K.; Bithell, E. G.; Tan, J.-C.; Cheetham, A. K. Structure and properties of an amorphous metal-organic framework. Phys. Rev. Lett. 2010, 104.

(25) Beake, E. O. R.; Dove, M. T.; Phillips, A. E.; Keen, D. A.; Tucker, M. G.; Goodwin, A. L.; Bennett, T. D.; Cheetham, A. K. Flexibility of zeolitic imidazolate framework structures studied by neutron total scattering and the reverse Monte Carlo method. J. Phys.: Condens. Matter 2013, 25, 395403.

(26) Keen, D. A.; Bennett, T. D. Structural investigations of amorphous metal-organic frameworks formed via different routes. Phys. Chem. Chem. Phys. 2018, 20, 7857-7861.

(27) Banlusan, K.; Antillon, E.; Strachan, A. Mechanisms of Plastic Deformation of MetalOrganic Framework-5. J. Phys. Chem. C 2015, 119, 25845-25852.

(28) Yang, Y.; Shin, Y. K.; Li, S.; Bennett, T. D.; van Duin, A. C. T.; Mauro, J. C. Enabling Computational Design of ZIFs Using ReaxFF. J. Phys. Chem. B 2018, 122, 9616-9624.

(29) Gaillac, R.; Pullumbi, P.; Coudert, F.-X. Melting of Zeolitic Imidazolate Frameworks with Different Topologies: Insight from First-Principles Molecular Dynamics. J. Phys. Chem. C 2018, 122, 6730-6736.

(30) Widmer, R. N. et al. Pressure promoted low-temperature melting of metal-organic frameworks. Nat. Mater. 2019, 18, 370-376.

(31) Vuilleumier, R.; Seitsonen, A. P.; Sator, N.; Guillot, B. Carbon dioxide in silicate melts at upper mantle conditions: Insights from atomistic simulations. Chemical Geology 2015, 418, $77-88$.

(32) Huang, D.; Badro, J.; Brodholt, J.; Li, Y. Ab Initio Molecular Dynamics Investigation of Molten Fe-Si-O in Earth's Core. Geophys. Res. Lett. 2019, 46, 6397-6405.

(33) Benoit, M.; Ispas, S.; Tuckerman, M. E. Structural properties of molten silicates from ab 
initio molecular-dynamics simulations: Comparison between $\mathrm{CaO}-\mathrm{Al}_{2} \mathrm{O}_{3}-\mathrm{SiO}_{2}$ and $\mathrm{SiO}_{2}$. Phys. Rev. B 2001, 64, 1044.

(34) Li, X.; Song, W.; Yang, K.; Krishnan, N. M. A.; Wang, B.; Smedskjaer, M. M.; Mauro, J. C.; Sant, G.; Balonis, M.; Bauchy, M. Cooling rate effects in sodium silicate glasses: Bridging the gap between molecular dynamics simulations and experiments. The Journal of Chemical Physics 2017, 147, 074501.

(35) Karagiaridi, O.; Lalonde, M. B.; Bury, W.; Sarjeant, A. A.; Farha, O. K.; Hupp, J. T. Opening ZIF-8: A Catalytically Active Zeolitic Imidazolate Framework of Sodalite Topology with Unsubstituted Linkers. J. Am. Chem. Soc. 2012, 134, 18790-18796.

(36) Park, K. S.; Ni, Z.; Cote, A. P.; Choi, J. Y.; Huang, R.; Uribe-Romo, F. J.; Chae, H. K.; O'Keeffe, M.; Yaghi, O. M. Exceptional chemical and thermal stability of zeolitic imidazolate frameworks. Proc. Natl. Acad. Sci. 2006, 103, 10186-10191.

(37) Soules, T. F. A molecular dynamic calculation of the structure of sodium silicate glasses. $J$. Chem. Phys. 1979, 71, 4570-4578.

(38) Robinson, K.; Gibbs, G. V.; Ribbe, P. H. Quadratic Elongation: A Quantitative Measure of Distortion in Coordination Polyhedra. Science 1971, 172, 567-570.

(39) Le Roux, S.; Jund, P. Ring statistics analysis of topological networks: New approach and application to amorphous $\mathrm{GeS}_{2}$ and $\mathrm{SiO}_{2}$ systems. Comput. Mater. Sci. 2010, 49, 70-83.

(40) Thornton, A. W.; Jelfs, K. E.; Konstas, K.; Doherty, C. M.; Hill, A. J.; Cheetham, A. K.; Bennett, T. D. Porosity in metal-organic framework glasses. Chem. Commun. 2016, 52, 37503753.

(41) Wang, Y.; Jin, H.; Ma, Q.; Mo, K.; Mao, H.; Feldhoff, A.; Cao, X.; Li, Y.; Pan, F.; Jiang, Z. A MOF Glass Membrane for Gas Separation. Angew. Chem. Int. Ed. 2020, 59, 4365-4369. 
(42) VandeVondele, J.; Krack, M.; Mohamed, F.; Parrinello, M.; Chassaing, T.; Hutter, J. Quickstep: fast and accurate density functional calculations using a mixed Gaussian and plane waves approach. Comput. Phys. Comm. 2005, 167, 103-128.

(43) Available online at http://www.cp2k.org.

(44) VandeVondele, J.; Krack, M.; Mohamed, F.; Parrinello, M.; Chassaing, T.; Hutter, J. Quickstep: Fast and accurate density functional calculations using a mixed Gaussian and plane waves approach. Comput. Phys. Comm. 2005, 167, 103-128.

(45) Bussi, G.; Donadio, D.; Parrinello, M. Canonical sampling through velocity rescaling. J. Chem. Phys. 2007, 126, 014101.

(46) Perdew, J. P.; Burke, K.; Ernzerhof, M. Generalized gradient approximation made simple. Phys. Rev. Lett. 1996, 77, 3865-3868.

(47) Grimme, S.; Antony, J.; Ehrlich, S.; Krieg, H. A consistent and accurate ab initio parametrization of density functional dispersion correction (DFT-D) for the 94 elements H-Pu. J. Chem. Phys. 2010, 132, 154104.

(48) Haigis, V.; Coudert, F.-X.; Vuilleumier, R.; Boutin, A. Investigation of structure and dynamics of the hydrated metal-organic framework MIL-53(Cr) using first-principles molecular dynamics. Phys. Chem. Chem. Phys. 2013, 15, 19049-19056.

(49) Goedecker, S.; Teter, M.; Hutter, J. Separable dual-space Gaussian pseudopotentials. Phys. Rev. B 1996, 54, 1703-1710.

(50) Pinheiro, M.; Martin, R. L.; Rycroft, C. H.; Jones, A.; Iglesia, E.; Haranczyk, M. Characterization and comparison of pore landscapes in crystalline porous materials. J. Mol. Graph. Model. 44, 208-219.

(51) Martin, R. L.; Smit, B.; Haranczyk, M. Addressing challenges of identifying geometrically diverse sets of crystalline porous materials. J. Chem. Inf. Model. 2012, 52, 308-318. 
(52) Willems, T. F.; Rycroft, C. H.; Kazi, M.; Meza, J. C.; Haranczyk, M. Algorithms and tools for high-throughput geometry-based analysis of crystalline porous materials. Micro. Meso. Mater. 149, 134-141. 